\title{
Accelerating AES with Vector Permute Instructions
}

\author{
Mike Hamburg \\ Computer Science Dept., Stanford University \\ mhamburg@cs.stanford.edu
}

\begin{abstract}
We demonstrate new techniques to speed up the Rijndael (AES) block cipher using vector permute instructions. Because these techniques avoid data- and key-dependent branches and memory references, they are immune to known timing attacks. This is the first constant-time software implementation of AES which is efficient for sequential modes of operation. This work can be adapted to several other primitives using the AES S-box such as the stream cipher LEX, the block cipher Camellia and the hash function Fugue. We focus on Intel's SSSE3 and Motorola's Altivec, but our techniques can be adapted to other systems with vector permute instructions, such as the IBM Xenon and Cell processors, the ARM Cortex series and the forthcoming AMD "Bulldozer" core.
\end{abstract}

Keywords: AES, AltiVec, SSSE3, vector permute, composite fields, cache-timing attacks, fast implementations.

\section{Introduction}

Since the 2001 selection of the Rijndael block cipher [6 as the Advanced Encryption Standard (AES), optimization of this cipher in hardware and in software has become a topic of significant interest.

Unfortunately, fast implementations of AES in software usually depend on a large table - 4kiB in the most common implementation - to perform the S-box and the round mixing function. While the table's size is problematic only on the most resource-constrained embedded platforms, the fact that the lookups are key- and data-dependent leads to potential vulnerabilities [2]. In an extreme case, Osvik et al. demonstrate how to extract the key from the Linux dm-crypt encrypted disk implementation with 65 milliseconds of measurements and 3 seconds of analysis [11.

This weakness seems to be intrinsic to the Rijndael algorithm itself. Except in bit-sliced designs, no known technique for computing the S-box is remotely competitive with table lookups on most processors, so that constant-time implementations are many times slower than table-based ones except in parallelizable modes of operation. Despite this issue, the Rijndael S-box' excellent cryptographic properties have led to its inclusion in other ciphers, including the LEX stream cipher [5], the Fugue hash function [7] and the Camellia block cipher [10].

C. Clavier and K. Gaj (Eds.): CHES 2009, LNCS 5747, pp. 18 32, 2009.

(C) International Association for Cryptologic Research 2009 
Several processors — including the VIA C3 and higher, the AMD Geode LX and the forthcoming Intel "Sandy Bridge" and AMD "Bulldozer" cores support hardware acceleration of Rijndael. This acceleration both speeds up the cipher and reduces its vulnerability to timing attacks. However, such hardware accelerators are processor-specific, and may not be useful in accelerating and protecting Fugue, Camellia or LEX.

We examine another hardware option for accelerating and protecting Rijndael: vector units with permutation instructions, such as the PowerPC AltiVec unit or Intel processors supporting the SSSE3 instruction set. Such units allow us to implement small, constant-time lookups with considerable parallelism. These vector units have attracted attention from AES implementors before: Bhaskar et al. considered a permutation-based implementation in 2001 4, and Osvik et al. mention such an implementation as a possible defense against cache-based attacks [1].

To implement the S-box, we take advantage of its algebraic structure using composite-field arithmetic 12 , that is, by writing $\mathbb{F}_{2^{8}}$ as a degree-2 field extension of $\mathbb{F}_{2^{4}}$. This allows efficient computation of the AES S-box without a large lookup table, and so is commonly used in hardware implementations of AES [14]. Käsper and Schwabe's software implementation in 8 takes this approach in a bit-sliced software implementation; this implementation holds the current PC processor speed record of 7.08 cycles/byte on the Intel Core i7 920 ("Nehalem") processor. Our technique achieves fewer cycles/byte on the PowerPC G4, but not on Intel processors.

As usual, hardware-specific optimizations are necessary to achieve optimal performance. This paper focuses on the PowerPC G4 1 and Intel Core i7 920 "Nehalem", but the techniques can be used in other processors. To this end, we demonstrate techniques that are not optimal on the G4e or Nehalem, but might be preferable on other processors.

\section{Preliminaries}

\section{$2.1 \quad$ Notation}

Because we are working with fields of characteristic 2, addition of field elements amounts to a bitwise exclusive or. We will still write it as "+".

Over subfields of $\mathbb{F}_{2^{8}}$, we will write $x / y$ for $x y^{254}$, which are equal when $y \neq 0$ because $y^{255}=1$. This extension of $\cdot / \cdot$ adds some corner cases when dividing by 0 . We will note such corner cases as they arise, and write $\approx$ instead of $=$ for formulae which are incorrect due to these corner cases.

The most frequent remedy for division by zero will be to set an "infinity flag". When dividing by a number with the infinity flag set, we will return 0 instead of the normal value. The flag is bit 4 on AltiVec and bit 7 in SSSE3, for simplicity

\footnotetext{
1 "G4e" is an unofficial designation of the PowerPC $744 \mathrm{x}$ and $745 \mathrm{x}$ G4 processors, commonly used to distinguish them from the earlier and considerably different line of G4 processors, the PowerPC 7400 and 7410.
} 
we simply set all 4 high bits. On AltiVec, use of an infinity flag requires extra masking to prevent the high bits of the input from interfering with the flag; in SSSE3, this masking is required anyway.

We write $a \| b$ for the concatenation of $a$ and $b$.

If $\boldsymbol{v}$ is a vector, then $v_{i}$ is its $i$ th component. By $\langle f(i)\rangle_{i=0}^{15}$, we mean the 16 element vector whose $i$ th element is $f(i)$. For example, if $\boldsymbol{v}$ has 16 elements, then $\boldsymbol{v}=\left\langle v_{i}\right\rangle_{i=0}^{15}$.

We number bits from the right, so that bit 0 is the $2^{0}$ place, bit 1 is the $2^{1}$ place, and so on.

\subsection{The Galois Fields $\mathbb{F}_{2^{8}}$ and $\mathbb{F}_{2^{4}}$}

AES is expressed in terms of operations on the Galois field $\mathbb{F}_{2^{8}}$. This field is written as

$$
\mathbb{F}_{2^{8}} \cong \mathbb{F}_{2}[x] /\left(x^{8}+x^{4}+x^{3}+x+1\right)
$$

When we write a number in hexadecimal notation, we mean to use this representation of $\mathbb{F}_{2^{8}}$. For example, 0x63 $=x^{6}+x^{5}+x+1$.

Because $\mathbb{F}_{2^{8}}$ is too large for convenient multiplication and division using AltiVec, we will work with the field $\mathbb{F}_{2^{4}}$, which we will write cyclotomically:

$$
\mathbb{F}_{2^{4}} \cong \mathbb{F}_{2}[\zeta] /\left(\zeta^{4}+\zeta^{3}+\zeta^{2}+\zeta+1\right)
$$

For this generator $\zeta$, we will express $\mathbb{F}_{2^{8}}$ as

$$
\mathbb{F}_{2^{8}} \cong \mathbb{F}_{2^{4}}[t] /\left(t^{2}+t+\zeta\right)
$$

The obvious way to represent elements of $\mathbb{F}_{2^{8}}$ is as $a+b t$ where $a, b \in \mathbb{F}_{2^{4}}$. A more symmetric, and for our purposes more convenient, representation is to set $\bar{t}:=t+1$ to be the other root of $t^{2}+t+\zeta$, so that $t+\bar{t}=1$ and $t \bar{t}=\zeta$. Then we may write elements of $\mathbb{F}_{2^{8}}$ uniquely as $x t+y \bar{t}$ with $x, y \in \mathbb{F}_{2^{4}}$ (here $y=a$ and $x=a+b$ from above). We will use these representations throughout this paper, and they will be reflected at the bit level: our implementations will compute with either $x \| y$ or $y \|(x+y)$, depending on timing constraints.

\subsection{AltiVec and the PowerPC G4}

We implemented AES on the PowerPC G4's AltiVec SIMD architecture, specifically the PowerPC 7447a G4e. We will be treating its 128-bit vectors as vectors of 16 bytes. In addition to bytewise arithmetic instructions, this processor has a vector permute instruction:

$$
\operatorname{vperm}(\boldsymbol{a}, \boldsymbol{b}, \boldsymbol{c}):=\left\langle(\boldsymbol{a} \| \boldsymbol{b})_{c_{i} \bmod 32}\right\rangle_{i=0}^{15}
$$

That is, $\operatorname{vperm}(\boldsymbol{a}, \boldsymbol{b}, \boldsymbol{c})$ replaces each element $c_{i}$ of $\boldsymbol{c}$ with the element of the concatenation of $\boldsymbol{a}$ and $\boldsymbol{b}$ indexed by $c_{i}$ 's 5 low-order bits.

We will find two uses for vperm. The first is to permute the block for the ShiftRows and MixColumns steps of AES. In this case, $\boldsymbol{c}$ is a fixed permutation 
Table 1. Intel SSE configurations

\begin{tabular}{rcccc}
\hline Core & SSE units & pshufb units & pshufb throughput & pshufb latency \\
\hline Conroe & 3 & 1 & 2 cycles & 3 cycles \\
Harpertown & 3 & 1 & 1 cycle & 1 cycle \\
Nehalem & 3 & 2 & 1 cycle & 1 cycle \\
\hline
\end{tabular}

and $\boldsymbol{a}=\boldsymbol{b}$ is the input block. The second use is 16 simultaneous lookups in a 32-element table, or a 16-element lookup table when $\boldsymbol{a}=\boldsymbol{b}$.

The processor can dispatch and execute any two vector instructions of different types 2 per cycle, plus a load or store. The arithmetic operations that we will use have a 1-cycle effective latency, and the permute operations have a 2cycle effective latency; both types have a 1/cycle throughput. Because we won't be saturating either the dispatcher or the load-store unit, loads and stores are effectively free in moderation.

\subsection{Intel SSSE3}

Intel's SSSE3 instruction set includes a weaker vector permute operation called pshufb. It differs from vperm in three ways. First, it only implements a 16-way shuffle, implicitly taking $\boldsymbol{a}=\boldsymbol{b}$. Second, if the highest-order bit of $c_{i}$ is set, then the $i$ th output will be 0 instead of $\boldsymbol{a}_{c_{i}} \bmod 16$. This is useful for implementing an infinity flag. Third, its operands follow a CISC 2-operand convention: its destination register is always the same register as $\boldsymbol{a}$, but $\boldsymbol{c}$ can be loaded from memory instead of from a register.

We will show benchmarks on three different Intel processors: a Core 2 Duo L7600 "Conroe", a Xeon E5420 "Harpertown" and a Core i7 920 "Nehalem". These processors have much more complicated pipelines than the G4e. All three can execute up to 3 instructions per cycle, all of which can be SSE logical instructions. Their shuffle units have different configurations as shown in Table 1 [.

\subsection{Log Tables}

Implementing multiplication and division with log tables is a well-known technique. However, it is not trivial to apply it in SIMD. AltiVec's vperm instruction only uses the low-order 5 bits of the permutation, so we must ensure that these 5 bits suffice to determine the result. Furthermore, when dividing we may wish to distinguish between $0 / 1,0 / 0$ and $1 / 0$ in order to implement an infinity bit. Within these constraints, we worked out the following tables largely by trial and error.

\footnotetext{
${ }^{2}$ There are 4 types of vector operations: floating-point operations; simple integer operations; complex integer operations such as multiplies; and permutations including whole-vector shifts, repacks and immediate loads.
} 
For log tables over $\mathbb{F}_{2^{4}}$, we set

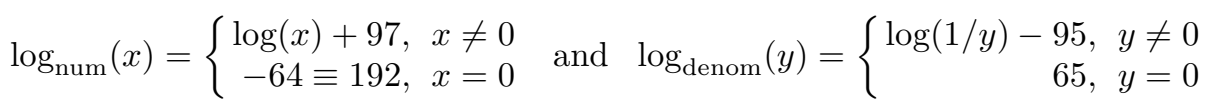

For multiplication, we perform an unsigned addition with saturation, defined as $a \uplus b:=\min (a+b, 255)$ so that

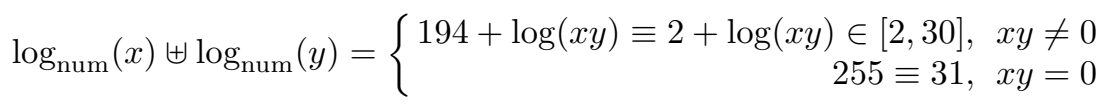

For division, we perform a signed addition with saturation, defined as $a \oplus b:=$ $\min (\max (a+b,-128), 127)$ so that

$$
\log _{\text {num }}(x) \oplus \log _{\text {denom }}(y)=\left\{\begin{aligned}
-128 \equiv 0, & x=0 \neq y \\
1, & x=0=y \\
2+\log (x / y) \in[2,30], & x \neq 0 \neq y \\
127 \equiv 31, & x \neq 0=y
\end{aligned}\right.
$$

Because these sums' residues mod 32 depend only on $x y$ or $x / y$ (and in the same way for both), a lookup table on the output can extract $x y$ or $x / y$. Furthermore, these $\log$ tables allow us to distinguish between $0 / 1,1 / 0$ and $0 / 0$.

\subsection{Cubic Multiplication}

Because pshufb operates on tables of size at most 16, it does not appear to admit a efficient implementation of log tables. It would be desirable to multiply instead using the "quarter-squares" identity $x y=(x+y)^{2} / 4-(x-y)^{2} / 4$. Unfortunately, this identity does not work over fields of characteristic 2 . We can instead set $\omega$ to a cube root of unity (so that $\omega^{2}=\omega+1$ ) and use an "omega-cubes" formula such as

$$
x y^{2}=\omega(x+\omega y)^{3}+\omega^{2}(\omega x+y)^{3}+\left(\omega^{2} x+\omega^{2} y\right)^{3}
$$

which is not as horrible as it looks because the map $(x, y) \rightarrow(x+\omega \sqrt{y}, \omega x+\sqrt{y})$ is linear. If $x$ and $y$ are given in this basis, $x y$ can be computed with 3 table lookups and 3 xors, but transforming into and out of this basis will cost 4-6 instructions. Alternatively, the above formula can be used to compute $x / y^{2}$ and $x^{2} / y$ in the high and low nibbles of a single register, but the lack of room for an infinity flag makes this strategy less useful.

On the processors we studied, cubic multiplication does not appear to be optimal technique for implementing AES, but it might be useful in other algorithms.

\section{Implementing Inversion}

This section deals with algorithms for inverting an element $x t+y \bar{t}$ of $\mathbb{F}_{2^{8}}$. 


\subsection{Classical Inversion}

The simplest way to compute $1 /(x t+y \bar{t})$ is to rationalize the denominator: multiplying top and bottom by $x \bar{t}+y t$ gives

$$
\frac{1}{x t+y \bar{t}}=\frac{x \bar{t}+y t}{x^{2} t \bar{t}+x y t^{2}+x y \bar{t}^{2}+y^{2} t \bar{t}}=\frac{x \bar{t}+y t}{x y+\left(x^{2}+y^{2}\right) \zeta}=\frac{x \bar{t}+y t}{(\sqrt{x y / \zeta}+x+y)^{2} \zeta}
$$

This last, more complicated expression is how we actually perform the computation: the multiplications are computed with log tables; the squares, square roots and multiplications and divisions by $\zeta$ come for free. This technique requires few operations, but it has many lookups on the critical path. Therefore it is optimal on the G4e in parallel modes, but not in sequential modes.

\subsection{Symmetric Inversion}

To improve parallelism, we can rearrange the above to the near-formula:

$$
\frac{1}{x t+y \bar{t}} \approx \frac{t}{x+\zeta(x+y)^{2} / y}+\frac{\bar{t}}{y+\zeta(x+y)^{2} / x}
$$

This formula is incorrect when $x=0$ or $y=0$, but this is easily fixed using an infinity flag. Since this technique can be computed in parallel, it is faster than classical inversion in sequential modes.

\subsection{Nested Inversion}

The most efficient formula that we found for Intel processors uses the fact that

$$
\frac{1}{1 / x+1 / \zeta(x+y)}+y \approx \frac{x y+\zeta\left(x^{2}+y^{2}\right)}{(1+\zeta) x+\zeta y}
$$

which leads to the monstrous near-formula

$$
\frac{1}{x t+y \bar{t}} \approx \frac{t+\zeta}{\frac{1}{1 / y+1 / \zeta(x+y)}+x}+\frac{\bar{t}+\zeta}{\frac{1}{1 / x+1 / \zeta(x+y)}+y}
$$

Division by zero is handled by using an infinity flag, which remarkably makes this formula correct in all cases. This technique performs comparably to symmetric inversion on the G443 , but much better on Intel because it does not require multiplication.

Figure 1 compares the parallelism of nested inversion and classical inversion. This diagram omits setup and architectural details, but is nonetheless representative: nested inversion completes faster despite having more instructions.

\footnotetext{
${ }^{3}$ In fact, their code is almost exactly the same. Nested inversion has different (and fewer) lookup tables, and some xors replacing adds, but its dependencies are identical. It is due entirely to momentum that our G4e implementation uses symmetric inversion.
} 

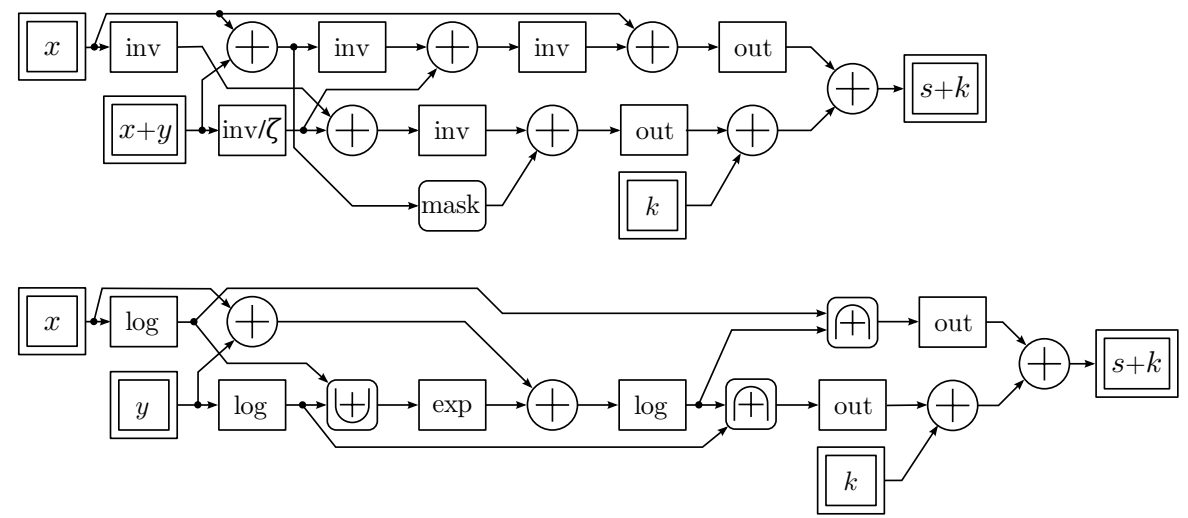

Fig. 1. Nested inversion (top) has more parallelism than classical inversion (bottom)

\subsection{Factored Inversion}

Another approach is to separate the variables, for example:

$$
\frac{1}{x t+y \bar{t}} \approx \frac{1}{x} \cdot \frac{1}{t+(y / x) \bar{t}}
$$

Once we compute $\log (y / x)$, we can compute (the $\log$ of) the right term in the form $a t+b \bar{t}$ with a pair of lookups. The formula is wrong when $x=0$, but we can look up a correction for this case in parallel with the rest of the computation. This technique combines the low latency of symmetric inversion with the high throughput of classical inversion. However, its many different lookup tables cause register pressure, so we prefer to use the more specialized formulas above.

\subsection{Brute Force}

The implementation in [4] uses a brute-force technique: a lookup in each of 8 tables of size 32 can emulate a lookup in a table of size 256 . This technique is less efficient than symmetric or nested inversion on all the processors we tested. For example, nested inversion requires 7 lookups into 4 tables of size 16 (with an infinity flag) and 6 xors.

\section{Implementing AES}

\subsection{The S-Box and the Multiplication by $0 \times 02$}

Every inversion algorithm described above (other than brute force) ends by computing $f(a)+g(b)$ for some $(f, g, a, b)$ using two shuffles and an xor. Therefore the S-box's linear skew can be folded into the tables for $f$ and $g$. However, the use of infinity flags (which may force a lookup to return 0) prevents folding in the addition. Therefore, we use tables for skew $(f(a))$ and skew $(g(b))$; we add $0 \times 63$ 
to the key schedule instead. Similarly, we can multiply by $0 x 02$ by computing $2 \operatorname{skew}(f(a))+2 \operatorname{skew}(g(b))$.

On the G4e, we make another modification to accomodate classical inversion. It happens that in the basis we use for classical inversion, skew $(a t)$ and skew $(b \bar{t})$ are functions of the low-order 4 bits of $0 \times 2 \cdot \operatorname{skew}(a t)$ and $0 \times 2 \cdot \operatorname{skew}(b \bar{t})$ but not vice-versa. As a result, we use fewer registers if we compute $0 x 2 \cdot$ skew $(a t)$ first.

\subsection{ShiftRows and MixColumns}

We have three options to perform the ShiftRows step and the MixColumns rotations.

1. We could keep AES' natural alignment, with each column in 4 contiguous bytes. This would allow us to use an unmodified key schedule. On the G4e, this technique makes MixColumns fast at the expense of ShiftRows. On Intel, both require permutations.

2. We could align each row into 4 contiguous bytes. On the G4, this makes ShiftRows fast at the expense of MixColumns, but relieves register pressure. On Intel, it allows the use of pshufd for MixColumns, but the lack of SIMD rotations means that ShiftRows requires a permutation. Also, an both an input and an output permutation are required.

3. We could use permutations for the MixColumns step. We conjugate by the ShiftRows permutation, so we need not physically perform ShiftRows at all. There will be a different forward and backward permutation each round, with a period of 4 rounds. For 128- and 256-bit keys, the number of rounds is not a multiple of 4 , so this technique requires either an input or an output permutation, but not both. (With the MixColumns technique used for classical inversion on the G4e, this method will always require an input or output permutation.) This technique seems to be the fastest both on the G4e and on Intel.

In any case, it is not advantageous to compute ShiftRows directly before computing MixColumns, because there are at least 2 registers live at all times. If ShiftRows is to be physically computed at all, this should be done at the beginning or end of the round, when only 1 register is live.

Let $r_{k}$ denote a left rotation by $k$ elements. To compute MixColumns, we compute $\boldsymbol{x}:=(a, b, c, d)$ and $0 \mathrm{x02} \cdot \boldsymbol{x}$ as above. We compute

$$
\boldsymbol{y}:=r_{1}(\boldsymbol{x})+0 \mathrm{x} 02 \cdot \boldsymbol{x}=(0 \mathrm{x} 02 \cdot a+b, 0 \mathrm{x} 02 \cdot b+c, 0 \times 02 \cdot c+d, 0 \times 02 \cdot d+a)
$$

We then compute the desired output

$$
r_{1}(\boldsymbol{y})+\boldsymbol{y}+r_{3}(\boldsymbol{x})=(0 \mathrm{x} 02 \cdot a+0 \mathrm{x} 03 \cdot b+c+d, \ldots)
$$

When using classical inversion, we compute $0 \times 02 \cdot \boldsymbol{x}$ before $\boldsymbol{x}$, so we use a similar addition chain that rotates $0 \mathrm{x} 02 \cdot \boldsymbol{x}$ first. 


\subsection{AddRoundKey and the Modified Key Schedule}

Naïvely we would add the round key either at the end of the round, or just before the end of the round, while waiting for a MixColumns permutation to finish executing. However, for some implementations, it is more convenient to add the round key during the S-box. Since every implementation of the S-box other than brute force ends with $f(a)+g(b)$ for some $(f, g, a, b)$, we can compute $f(a)+k^{\prime}+g(b)$, with the addition of $k^{\prime}$ in parallel with the computation of $g(b)$. This technique is more efficient on Intel and on the original G4, while performing the same on the G4e.

However, this trick makes the key schedule more complicated: the vector $\boldsymbol{k}^{\prime}$ will be multiplied by

$$
M:=\left(\begin{array}{llll}
0 & 1 & 1 & 1 \\
1 & 0 & 1 & 1 \\
1 & 1 & 0 & 1 \\
1 & 1 & 1 & 0
\end{array}\right)
$$

during MixColumns. Since $M$ is its own inverse, this means that we should set $\boldsymbol{k}^{\prime}=\boldsymbol{M} \boldsymbol{k}$. As a result, this trick may not be desirable when very high key agility is required.

Our key schedule also differs from the standard schedule in that keys must be transformed into the $(t, \bar{t})$ basis for $\mathbb{F}_{2^{8}}$ and rotated by the ShiftRows permutation.

\section{$5 \quad$ Parallelism and Related Optimizations}

\subsection{Interleaving}

On the G4e, the classical inversion algorithm gives few instructions, but a high latency. What is more, the instructions are balanced: they put an equal toll on the simple integer and permute units. As a result, they interleave very well: we can run 2 rounds in 24 cycles, compared to 1 round in 20 cycles using classical inversion or 17 cycles using symmetric inversion. For modes which allow parallel encryption, this boosts encryption speed by about $40 \%$. Similiarly, we can interleave 4 rounds in 46 cycles, which should boost encryption speed by another $4 \%$ or so.

\subsection{Byte-Slicing}

A more extreme transformation is to byte-slice encryption. This technique performs 16 encryptions in parallel. Instead of holding state for each of the 16 bytes in one encryption, a vector holds state for one byte in each of the 16 different encryptions. This allows two speedups. First, since each byte is in a separate register, no permutations are needed for ShiftRows and MixColumns. Second, the same key byte is added to every position of the vector. As a result, we can simply add this key byte to the exponentiation tables each round. Of course, to 
add this byte every round would save no time at all, but if we schedule each round's exponentiation table ahead of time, we will save an xor instruction.

We implemented this technique on the G4e, and found two difficulties. One is that we must split the data to be encrypted into byte-sliced form, and merge it back into ordinary form at the end of the encryption, which costs about $1 / 8$ cycle per byte in each direction. A second problem is that the byte-sliced round function becomes limited by integer operations, so that saving permutations doesn't help. To alleviate this problem, we can replace a vsrb (element shift right) instruction with a vsr (whole-vector shift right) instruction, which uses the permutation unit on the G4e. In conjunction with double-triple mixing, this optimization reduces the cipher to 9.5 cycles per main round and 6.5 cycles for the final round.

Conveniently, Rogaway et al's OCB mode for authenticated encryption with associated data [13] is simpler in byte-sliced form. The offset $\Delta$ may be stored for each slice; then most of the multiplication by $2^{16}$ - that is, shifting left by 2 bytes - consists of renumbering slice $n$ as slice $n-2$. The lowest three slices will be a linear function of the upper two slices and the lowest slice; this can be computed with two shifts, 8 permutations and 6 xors. This representation is highly redundant; it is possible that a more concise representation would allow a more efficient computation of the OCB offset $\Delta$.

\subsection{Double-Triple Mixing}

Let $(a, b, c, d)$ be the output of the S-box. Our implementation of mixing for standard round functions computed $2 a$ then $a$. For highly parallel modes, we can do better by computing $2 a$ and then $3 a$. If we let

$$
(\alpha, \beta, \gamma, \delta):=(3 a+2 b, 3 b+2 c, 3 c+2 d, 3 d+2 a)
$$

then the output of the mixing function is

$$
(\beta+\gamma+\delta, \alpha+\gamma+\delta, \alpha+\beta+\delta, \alpha+\beta+\gamma)
$$

This output is easily computed using 10 xors instead of 12 . The technique we use computes

$$
\begin{aligned}
& c_{0}:=\alpha, \quad c_{1}:=\alpha+\beta, \quad c_{2}:=\alpha+\beta+\gamma, \quad c_{3}:=\alpha+\beta+\delta, \\
& c_{4}:=\beta+\gamma=c_{2}+c_{0} \\
& c_{5}:=\alpha+\gamma+\delta=c_{3}+c_{4} \\
& c_{6}:=\beta+\gamma+\delta=c_{1}+c_{5}
\end{aligned}
$$

and outputs $\left(c_{6}, c_{5}, c_{3}, c_{2}\right)$. In addition to taking only 10 xors, this method takes its inputs in order $(\alpha, \beta, \gamma, \delta)$ and immediately xors something into them. These features lead to significant savings in register usage, latency and complexity. We suspect that the savings would be even better on Intel hardware. 


\subsection{Counter-Mode Caching}

Bernstein and Schwabe [3 call attention to a useful optimization in Hongjun Wu's eStream implementation of counter mode. Except in every 256th block, only the last byte of the input changes. As a result, only one S-box needs to be computed the first round. Its output affects only 4 bytes, so only 4 S-boxes need to be computed the second round. In a 10-round, 128-bit AES encryption, this saves about 1.7 rounds on average, or about $17 \%$ (slightly more, because the last round is shorter). What is more, it allows us to avoid transforming the input into byte-sliced form.

\subsection{Scalar-Unit Assistance}

Following [4, we considered using the G4e's scalar unit to perform two of the vector xors, reducing the cipher to 9 cycles per round. However, the expense of shuttling data between the vector and scalar units nullifies any advantage from this technique.

\section{Decryption}

Decryption is more difficult than encryption, because the MixColumns step is more complicated: the coefficients are (0xOE, 0x09, OxOD, OxOB), which are linearly independent over $\mathbb{F}_{2}$. As a result, all four coefficients must be looked up separately. This requires 4 more tables than encryption (minus one for permutations, because we can use only forward permutations with this method), and on Intel means that the lookup tables spill to memory.

\section{$7 \quad$ Benchmarks}

We initially tested several experimental implementations on the G4e. They include heavily optimized implementations of several modes several modes, but are also somewhat incomplete; in particular, we did not implement encryption of unaligned data or any sort of decryption.

After realizing that the same techniques are applicable to Intel using SSSE3, we set out to build a practical AES library for Intel machines. However, optimization on $\mathrm{x} 86$ processors is much more difficult than on the PowerPC, so our library does not yet approach its theoretical maximum performance. Our Intel library also does not yet implement as many modes or techniques, but it does implement encryption and decryption on aligned and unaligned data.

We tested our implementation on four machines, whose specifications are listed in Table 2 ,

Our byte-sliced implementations are experimental and so far incomplete. We benchmarked each individual component of the algorithm and added together the times. Similarly, on the G4e we benchmarked only the streaming steps of OCB mode, not the nonce generation and finalization. These consist of one 
Table 2. Bechmark machine specifications

\begin{tabular}{llll}
\hline Machine & Processor & Core & Speed \\
\hline altacaca & Motorola PowerPC G4 7447a & Apollo 7 & $1.67 \mathrm{GHz}$ \\
peppercorn & Intel Core 2 Duo L7500 & Conroe & $1.60 \mathrm{GHz}$ \\
WhisperMoon & Intel Xeon E5420 & Harpertown & $2.50 \mathrm{GHz}$ \\
lahmi & Intel Core i7 920 & Nehalem & $2.67 \mathrm{GHz}$ \\
\hline
\end{tabular}

Table 3. Encryption timings on altacaca in cycles per byte

\begin{tabular}{rlllrrrrrrrr}
\hline Implementation & Par & Mode & \multicolumn{3}{c}{ 128-bit key } & \multicolumn{3}{c}{ 192-bit key } & \multicolumn{3}{c}{ 256-bit key } \\
& & & 32 & 512 & long & 32 & 512 & long & 32 & 512 & long \\
\hline Symmetric & 1 & ECB & 11.3 & 10.6 & 10.7 & 14.1 & 12.7 & 12.8 & 17.0 & 14.8 & 14.9 \\
& 1 & CBC & 11.3 & 10.8 & 10.8 & 14.1 & 12.9 & 12.9 & 17.0 & 15.0 & 15.1 \\
Classical & 2 & ECB & 8.5 & 7.9 & 7.8 & 11.3 & 9.4 & 9.3 & 11.3 & 10.8 & 10.8 \\
& 2 & CTR & 9.4 & 7.9 & 7.9 & 11.3 & 9.4 & 9.3 & 11.3 & 10.8 & 10.8 \\
& 2 & OCB & 19.5 & 8.6 & 7.8 & 25.4 & 10.2 & 9.3 & 25.4 & 12.0 & 10.8 \\
Classical (sliced) & 16 & CTR & & & 5.4 & & & 6.7 & & & 7.9 \\
& 16 & OCB & & & 6.6 & & & 7.8 & & & 9.0 \\
\hline \multirow{2}{*}{ openssl speed } & 1 & CBC & \multicolumn{3}{c}{32.6} & & 36.4 & & & 40.5 \\
\hline
\end{tabular}

encryption each, so we have added the time required for two encryptions. We expect that a complete implementation would be slightly more efficient due to function call overhead.

We tested encryption and decryption on messages of size 32, 512 and 4096 bytes, with 128-, 192- and 256-bit keys.

Our classical encryption code was optimized for OCB mode; we expect that its ECB and CTR timings could be improved by 1-2\% with further tuning. Due to cache effects, encryption of long messages is slightly slower than encryption of short messages in some cases.

\subsection{Architecture-Specific Details}

Alignment. We tested with 16-byte-aligned input, output and key. Our Intel code supports unaligned input and output; our G4e code does not. Both implementations require 16-byte-aligned round keys, but this is enforced by our library. Our code currently only supports messages which are an integral number of blocks; we are intending to change this before release.

Loop unrolling. We did not unroll the round function at all, except in the bytesliced case, in which we unrolled it 4 ways. Experiments showed that unrolling was generally unnecessary for optimum performance on the G4e, and our Intel code is still largely unoptimized. 
Table 4. Encryption timings on peppercorn in cycles per byte

\begin{tabular}{rlllllllllll}
\hline Implementation & \multirow{2}{*}{ Mode } & \multicolumn{3}{c}{ 128-bit key } & \multicolumn{3}{c}{ 192-bit key } & \multicolumn{3}{c}{ 256-bit key } \\
& & 32 & 512 & long & 32 & 512 & long & 32 & 512 & long \\
\hline \multirow{2}{*}{ Nested } & ECB & 22.0 & 21.6 & 21.5 & 26.3 & 25.3 & 25.5 & 30.6 & 29.9 & 30.1 \\
& $\mathrm{ECB}^{-1}$ & 27.0 & 26.5 & 26.3 & 32.3 & 31.7 & 31.4 & 37.8 & 37.1 & 37.0 \\
& $\mathrm{CBC}^{-1}$ & 22.3 & 21.6 & 21.4 & 26.5 & 25.8 & 25.6 & 31.0 & 30.0 & 30.0 \\
& $\mathrm{CBC}^{-1}$ & 27.4 & 26.3 & 25.9 & 32.4 & 31.7 & 31.4 & 37.8 & 37.3 & 36.9 \\
& $\mathrm{CTR}$ & 22.2 & 21.5 & 21.8 & 26.5 & 25.8 & 25.8 & 30.7 & 30.1 & 29.9 \\
& $\mathrm{OCB}^{-1}$ & 44.2 & 23.6 & 22.3 & 52.8 & 28.2 & 26.5 & 61.4 & 32.5 & 30.8 \\
& $\mathrm{OCB}^{-1}$ & 54.7 & 28.7 & 27.4 & 64.5 & 34.4 & 32.8 & 75.4 & 40.0 & 37.8 \\
\hline \multirow{2}{*}{ openssl speed } & $\mathrm{CBC}^{*}$ & & & 18.8 & & & 21.4 & & & & 24.1 \\
\hline
\end{tabular}

Table 5. Encryption timings on WhisperMoon in cycles per byte

\begin{tabular}{rlrrrrrrrrrr}
\hline Implementation & \multirow{2}{*}{ Mode } & \multicolumn{3}{c}{ 128-bit key } & \multicolumn{3}{c}{ 192-bit key } & \multicolumn{3}{c}{ 256-bit key } \\
& & 32 & 512 & long & 32 & 512 & long & 32 & 512 & long \\
\hline \multirow{2}{*}{ Nested } & ECB & 11.8 & 11.1 & 11.0 & 13.9 & 13.2 & 13.3 & 16.1 & 15.4 & 15.4 \\
& $\mathrm{ECB}^{-1}$ & 14.7 & 14.3 & 14.4 & 17.7 & 17.0 & 17.1 & 20.4 & 19.9 & 19.9 \\
& $\mathrm{CBC}^{-1}$ & 11.6 & 11.1 & 11.2 & 14.3 & 13.3 & 13.5 & 16.1 & 15.4 & 15.8 \\
& $\mathrm{CBC}^{-1}$ & 14.8 & 14.2 & 14.2 & 17.6 & 17.0 & 17.0 & 20.4 & 20.2 & 20.2 \\
& $\mathrm{CTR}$ & 11.9 & 11.1 & 11.1 & 14.1 & 13.3 & 13.4 & 16.4 & 15.4 & 15.8 \\
& $\mathrm{OCB}^{-1}$ & 23.3 & 12.3 & 11.7 & 27.6 & 14.5 & 13.7 & 31.8 & 17.0 & 16.1 \\
& $\mathrm{OCB}^{-1}$ & 29.4 & 15.4 & 14.6 & 35.0 & 18.5 & 17.5 & 40.9 & 21.5 & 20.4 \\
\hline \multirow{2}{*}{ openssl speed } & $\mathrm{CBC}$ & & & 18.7 & & & 21.3 & & & 23.9 \\
\hline
\end{tabular}

Table 6. Encryption timings on lahmi in cycles per byte

\begin{tabular}{rlrrrrrrrrr}
\hline Implementation & \multirow{2}{*}{ Mode } & \multicolumn{3}{c}{ 128-bit key } & \multicolumn{3}{c}{ 192-bit key } & \multicolumn{3}{c}{ 256-bit key } \\
& & 32 & 512 & long & 32 & 512 & long & 32 & 512 & long \\
\hline \multirow{2}{*}{ Nested } & $\mathrm{ECB}$ & 10.3 & 10.0 & 9.9 & 12.4 & 11.9 & 11.9 & 14.8 & 13.9 & 13.9 \\
& $\mathrm{ECB}^{-1}$ & 12.9 & 12.4 & 12.4 & 15.3 & 15.0 & 15.0 & 18.0 & 17.6 & 17.6 \\
& $\mathrm{CBC}^{-1}$ & 10.8 & 10.3 & 10.3 & 12.9 & 12.4 & 12.3 & 14.6 & 14.4 & 14.2 \\
& $\mathrm{CBC}^{-1}$ & 13.0 & 12.6 & 12.5 & 16.1 & 15.1 & 15.2 & 18.2 & 17.8 & 17.8 \\
& $\mathrm{CTR}$ & 10.4 & 10.0 & 10.0 & 12.4 & 12.0 & 11.9 & 14.2 & 13.9 & 13.9 \\
& $\mathrm{OCB}^{-1}$ & 21.4 & 11.1 & 10.5 & 25.5 & 13.2 & 12.5 & 29.5 & 15.3 & 14.5 \\
& $\mathrm{OCB}^{-1}$ & 26.4 & 13.8 & 13.1 & 31.4 & 16.5 & 15.6 & 36.6 & 19.2 & 18.2 \\
\hline openssl speed & $\mathrm{CBC}$ & & & 17.6 & & & 20.2 & & & 22.6 \\
\hline
\end{tabular}




\section{Other Processors}

Our techniques are applicable to the PowerPC e600 (modern, embedded G4) with essentially no modification. Other processors have different instruction sets, pipelines and numbers of registers, and so our techniques will require modification for optimimum implementation.

The earlier PowerPC 7410 (original G4) can only issue 2 instructions per cycle instead of three. Because we no longer have "free" loads and branches, more unrolling and caching is necessary. However, the 7410's vperm instruction has an effective latency for only 1 cycle. After accounting for these differences, performance should be slightly faster than on the 7447 when using symmetric inversion, and about the same speed when using interleaved classical inversion. As a result, the interleaved case is less desirable.

The PowerPC 970 (G5) has much higher instruction latencies than the G4, and penalties for moving data between functional units. As a result, more parallelism is required to extract reasonable performance from the G5. It is possible that brute force is the best way to compute the S-box, due to its very high parallelism.

The IBM Cell's SPEs have many more registers than the G4e. Furthermore, their spu_shuffle instruction differs from vperm in that it assigns a meaning to the top 3 bits of their input, so inputs need to be masked before permuting. Furthermore, the SPEs lack a vector byte add with saturation, so a different log-table technique needs to be used. For multiplication, we suggest mapping 0 to $0 \times 50$ and nonzero $x$ to $0 \times 30+\log x$, so that $\log (0$. $0) \rightarrow 0 \times A 0$ and $\log (0 \cdot x) \rightarrow[0 \times 80,0 \times 8 E]$, all of which code for $0 \times 00$ in the spu_shuffle instruction. We estimate that with a byte-sliced 128-bit implementation, a Cell SPU would require approximately 8 clock cycles per byte encrypted.

The forthcoming AMD "Bulldozer" core will feature an SSE5 vector permute instruction similar to AltiVec's. Like the Cell's spu_shuffle, this instruction assigns additional meaning to the 3 bits of the input field, which means that more masking and different log tables will be needed. SSE5 has fewer registers than AltiVec, but its ability to take arguments from memory instead of from registers may make up for this if the latency penalty is low enough.

ARM's NEON vector instruction set features a vector permute instruction, but its performance is significantly worse than SSSE3 or AltiVec. Nested inversion is probably the most practical technique due to its smaller tables.

\section{Conclusions and Future Work}

We have presented a technique for accelerating AES using vector permute units, while simultaneously thwarting known timing- and cache-based attacks. Our technique is the first software design to yield a fast, constant-time implementation for sequential modes of operation. Our results include some $150 \%$ 
improvement over current implementations on the G44. On recent x86-64 processors, it is some $41 \%$ slower than Käsper and Schwabe's bitsliced implementation 8, but doesn't require a parallel mode to attain this speed.

We expect that microarchitectural optimization can improve the speed of our code significantly. This will be a major focus of future work. We also expect that this work can be applied to other primitives; it would be interesting to see if Camellia, Fugue or LEX can be implemented as efficiently.

\section{References}

1. Intel 64 and ia-32 architectures optimization reference manual (2009)

2. Bernstein, D.: Cache-timing attacks on AES. Technical report (2005)

3. Bernstein, D.J., Schwabe, P.: New AES software speed records (2008)

4. Bhaskar, R., Dubey, P., Kumar, V., Rudra, A., Sharma, A.: Efficient Galois field arithmetic on SIMD architectures. In: Proceedings of the 15th ACM Symposium on Parallelism in Algorithms and Architectures, pp. 256-257 (2003)

5. Biryukov, A.: A new 128-bit-key stream cipher: LEX. In: eSTREAM, ECRYPT Stream Cipher Project, Report 2005/013 (2005)

6. Daemen, J., Rijmen, V.: Aes proposal: Rijndael (1999)

7. Halevi, S., Hall, W., Jutla, C.: The hash function fugue (2008)

8. Käsper, E., Schwabe, P.: Faster and timing-attack resistant aes-gcm. In: Clavier, C., Gaj, K. (eds.) CHES 2009. LNCS, vol. 5747, pp. 1-17. Springer, Heidelberg (2009)

9. Lipmaa, H.: AES ciphers: speed (2006)

10. Nakajima, J., Aoki, K., Kanda, M., Matsui, M., Moriai, S., Ichikawa, T., Tokita, T.: Camellia: A 128-bit block cipher suitable for multiple platforms - design and analysis (2000)

11. Osvik, D.A., Shamir, A., Tromer, E.: Cache attacks and countermeasures: the case of aes. In: Pointcheval, D. (ed.) CT-RSA 2006. LNCS, vol. 3860, pp. 1-20. Springer, Heidelberg (2006)

12. Rijmen, V.: Efficient implementation of the rijndael s-box (2000)

13. Rogaway, P.: Authenticated-encryption with associated-data. In: Proc. 9th CCS, pp. 98-107. ACM Press, New York (2002)

14. Rudra, A., Dubey, P.K., Jutla, C.S., Kumar, V., Rao, J.R., Rohatgi, P.: Efficient Rijndael encryption implementation with composite field arithmetic. In: Koç, Ç.K., Naccache, D., Paar, C. (eds.) CHES 2001. LNCS, vol. 2162, pp. 171-184. Springer, Heidelberg (2001)

\footnotetext{
${ }^{4}$ Bernstein and Schwabe claim 14.57 cycles/byte for CTR mode on a G4. We have not benchmarked their code on a G4e; Denis Ahrens claims a $4 \%$ speedup for AES on the G4e 9], so we estimate that Bernstein and Schwabe's code will also gain 4\% on the G4e.
} 